\title{
Channel State Information (CSI) based Sparse Reconstruction for Biomedical Applications Using hybrid mm-WAVE MIMO System
}

\author{
Sunil Kumar $\mathbf{M}^{1}$, Narayanappa $\mathrm{CK}^{2}$, M. Nagendra Kumar ${ }^{3}$ \\ ${ }^{1}$ Research Scholar, VTU Belagavi, Department of Medical Electronics, RIT, Bengaluru, Karnataka \\ ${ }^{2}$ Department of Medical Electronics \& Engineering, Ramaiah Institute of Technology, Bengaluru, Karnataka \\ ${ }^{3}$ Department of Electronics \& Communication Engg, SJC Institute of Technology, Chickballapur, India
}

Article History: Received: 10 November 2020; Revised: 12 January 2021; Accepted: 27 January 2021;

Published online: 05 April 2021

\begin{abstract}
The significance of Channel State Information (CSI) is very essential in a hybrid mm-WAVE Multiple Input Multiple Input (MIMO) System due to its direct dependency on medium capacity and energy efficiency of a network. Therefore, a Channel State Information (CSI)-based Sparse Reconstruction (CSISR) technique is adopted for effective evaluation of CSI for future $5 \mathrm{G}$ cellular network implementation. A hybrid mm-WAVE MIMO communication system is also employed for effective bandwidth spectrum utilization. Furthermore, a joint sparse coding algorithm is introduced to study the channel matrices of hybrid mm-WAVE MIMO system. The proposed CSISR technique ensure proficient signal reconstruction, signal compression and resource reduction by exploiting sparsity of channel matrix. The proposed CSISR technique under low SNR conditions as well for hybrid mm-WAVE MIMO system with optimization of pre-processors and combiners. The performance throughput of proposed CSISR technique is measured against conventional algorithms considering power consumption, Normalized Mean Square Error (NMSE) and spectral efficiency of the mm-Wave MIMO system. The superiority of proposed CSISR technique is concluded based on simulations considering different system configurations and performance matrices.
\end{abstract}

Keywords: Hybrid mm-WAVE MIMO System, Channel State Information, Sparse Reconstruction, Biomedical Applications, 5-th Generation (5G) Cellular Network

\section{Introduction}

Recent developments in several technologies have enormously benefitted sectors like Healthcare, Manufacturing, Telecom, Communication, sports and music based scientific assessment approaches as well [1]. However, healthcare devices and equipment requires constant technology developments in order to get maximum yield from their diagnosis efforts. Moreover, healthcare instruments, medical devices and diagnosis methods are directly dependent on technology developments. The exceptional developments in technology has made healthcare departments very powerful and efficient. Diagnosis methods like Magnetic Resonance Imaging (MRI), Respiratory Diagnosis, blood pressure measurement and Electrocardiograph (ECG) for electric movement recording of heart etc. become highly effective and successful due to the utilization of high-tech technologies. Furthermore, these diagnosis methods need high-tech reliable medical devices and instruments which works on scientific principles and signal processing. However, digital signal processing and these diagnosis methods generates enormous amount of information whose storage and transmission from one place to another is highly complex and challenging process.

Therefore, highly efficient compression methods are required to transmit these enormous amount of medical data which can ensure preservation of its essential features. Thus, several researchers have shown their interest in finding a suitable compression method for medical data transmission and many researchers have considered Compressive Sensing (CS) method is a suitable and attractive option for biomedical signal compression [2-5]. CS is an exceptional signal compression technique which exploits the sparsity nature of medical signals to attain real-time, precise and power-effective compression outcomes. CS technique can ensure complete data extraction from small set of compressed signals [6]. Thus, this technique can be efficiently utilized for the reconstruction of biomedical signals. Moreover, the difference between actual and reconstructed biomedical signals defines the minimum number of samples needed for effective signal reconstruction. The minimum number of samples needed for signal reconstruction in CS method is lower than the Nyquist rate.

Furthermore, biomedical signals can be defined as the analysis of physiological organism actions. Here, biomedical signal examination include analysis of whole body which can be conducted using various medical equipment and devices. Generally, biomedical signals acquired from this analysis possess enormous information which need to be transmitted through a communication link after compression. However, transmission of bulky medical information requires an implementation of superfast high-frequency high-bandwidth technology. Thus, several researches have shown Fifth generation $(5 \mathrm{G})$ cellular networks is a promising solution for high data transmission which provides excellent coverage with low as well as high frequencies for multi-radio access technologies and possess exceptional information rate up to many gigabits and ensure ultra-reliable services. 
From a recent survey it is predicted that traffic in mobile applications will enhance 1000 times while using future 5G network in comparison with current $4 \mathrm{G}$ network. Thus, future 5G network will need at least a bandwidth up to $100 \mathrm{MHz}$, utilization of greater than hundred antennas and ultra-densely deployed Source Station (SS) to support high data rate which is a quite challenging and complex task.

Therefore, several experts and researchers have identified utilization of Millimeter-wave (mm-Wave) frequency bands in future $5 \mathrm{G}$ technology as a most promising solution which ensures high-frequency highbandwidth spectrum. The Millimeter-wave (mm-Wave) technology makes future cellular network communication very powerful and efficient [7]. The mm-Wave communication supports high data rates such as gigabits-per-second which can be implement byexploiting huge bandwidth present at $\mathrm{mm}$-Wave frequencies. The bandwidth spectrum occupied by $\mathrm{mm}$-Wave communication is 200 times more than the spectrum available in current cellular networks. The multiple-input multiple-output (MIMO) technology can work in coordination with $\mathrm{mm}$-Wave communication to ensure large information rates, greater spectral efficiency, lower latency and greater ability to handle congestion. However, occurrence of large attenuation and signal absorption in an mmWave communication is a concerning area [8]. The utilization of several antenna array elements in mm-Wave communication reduces attenuation and signal absorption to a significant extent.

Several researchers have provided their attention for the enhancement of compression sensing and mm-Wave communication technology and some of them are presented in the following paragraph. In [9], mm-Wave communication technique is presented based on sparse encoding and decoding methods and beam alignment problem is discussed and sparse scattering nature is used for compressive phase-less measurements. In [10], a hybrid mm-WAVE MIMO System is introduced for the estimation of channel information using Off-Grid Dirichlet Kernels. Here, an Orthogonal Matching Pursuit (OMP) algorithm is utilized for efficiency enhancement. In [11], a research survey on mm-Wave user association mechanism is conducted to provide a comprehensive discussion over mm-Wave spectrum sharing methods, their challenges and how these issues can be avoided. In [12], a Hybrid Beamforming mm-Wave Systems is introduced for the estimation of channel information in accordance with Wideband MIMO using random spatial sampling. This technique focuses on channel information recovery based on the angular information and low rank property. However, there are several issues present in mm-Wave communication technology which need to be discussed comprehensively for the enhancement of spectrum efficiency such as high attenuation, severe path loss, signal propagation delay, high power consumption due to utilization of large antenna array elements, accurate estimation of channel state information acquired at transmitter and reception side, high computational cost, spectral efficiency loss in conventional methods, high overhead and optimization problem.

Therefore, sparse coding method is a promising solution for efficient information compression as well as for signal transmission. The sparse nature of $\mathrm{mm}$-Wave communication frequency bands can reduce beam alignment problem significantly. Sparse coding is represented as the signal processing scheme that focusses on retrieving a frame from few training samples in which sparse nature is present and it extracts sparse nature from few training samples by exploiting linear combination of basic elements. This method is primarily utilized for signal reconstruction, signal compression and resource reduction. The main objective of sparse coding method is effective signal measurement by finding knowledge about their signal structure. This method can help in storage capacity enhancement, fast transmission and effective compression without noise. However, Channel State Information (CSI) estimation and extraction become quite challenging due to the utilization of antenna array elements to a large extent and high channel inconsistency in mm-WAVE communication technology [12]. Channel State Information (CSI) is a representation of channel attributes of a communication network. CSI refers to the signal propagation information from transmitter to receiver and shows the mutual signal scattering, signal fading and power consumption effects. Moreover, CSI estimation is very crucial and essential for reliable data transmission with current channel conditions and with high information rates in mm-WAVE communication technology [13-14]. The efficiency of mm-WAVE communication technology is directly depends upon Channel State Information (CSI) of source station and receiver.

Therefore, a Channel State Information (CSI)-based Sparse Reconstruction (CSISR) technique is introduced for efficient estimation of CSI at both transmitter and receiver side to enhance efficiency of hybrid mm-WAVE communication system for future 5G cellular network. The proposed CSISR technique requires very few training samples for efficient extraction of CSI with low computational complexity. The proposed CSISR provide significant performance while signal reconstruction, signal compression and resource reduction due to the use of fewer training samples by exploiting sparse nature of biomedical signalsunder low SNR conditions as well. Furthermore, a joint sparse coding algorithm is presented for the optimization of transmitted and received biomedical signals and to study the channel matrices of hybrid mm-WAVE MIMO system. This algorithm jointly optimize the common sparse nature of subcarriers. The proposed CSISR technique ensure significant 
reduction in antenna disturbances and antenna coupling errors. The proposed CSISR technique make their efforts for avoiding optimization problem and reducing channel overhead present in the conventional orthogonal matching pursuit (OMP) algorithm. The performance of proposed sparse coding algorithm is compared with conventional algorithms in terms of SNR, NMSE and efficiency of the mm-Wave MIMO systems.

This paper is arranged in following manner which is described below. Section 2, discusses about the literature survey presented for mm-WAVE technology, their identified issues and how those issues can be sorted with the help of proposed Channel State Information (CSI)-based sparse reconstruction techniques. Section 3, describes about the methodology utilized in proposed CSI-based sparse reconstruction techniques. Section 4 discusses about experimental results and their comparison with state-of-arts-compression techniques and section 5 concludes the paper.

\section{Literature Survey}

Recent researches have shown Millimeter wave (mm-Wave) communication have tremendous potential to collaborate with future 5G cellular networks as future cellular networks will require bandwidth up to some GBPS according to several experts which cannot be achieved by current bandwidth spectrums. However, Millimeter wave (mm-Wave) communication frequency bands are immensely capable of delivering the desired bandwidth for effective implementation of future $5 \mathrm{G}$ cellular networks. Though, there are a significant research gap present for the efficient utilization of mm-Wave communication frequency bands. There are several challenges are encountered in mm-Wave communication which can degrade their efficiency and need to be formulated very quickly to avoid any future complexities as the market expectations and demands are rapidly growing. Some of the challenges are high power consumption, accurate estimation of channel state information, high computational cost, spectral efficiency loss, optimization problem and high channel overhead. Therefore, many researchers are making their efforts to handle these challenges and enhance spectrum efficiency. Some of the literatures are presented below in the following paragraph.

In [15], a Millimeter wave (mm-Wave) communication system is employed for the estimation of channel sparsity using block sparse nature. Moreover, low-rank structures are utilized for Doppler shifts and optimize their parameters. These methods are utilized for effective reconstruction of signals by exploiting channel sparsity. In [16], a low-rank matrix completion (LRMC) survey is conducted for understanding challenges and potentials of LMRC technique which can be widely utilized in applications such as image compression, image reconstruction, Massive multiple-input multiple-output and localization in IoT devices etc. Convolutional Neural Network (CNN) is also adopted for exploiting the graph structure of LMRC technique. In [17], mm-Wave Massive MIMO System is introduced to reduce computational complexity of the network using Orthogonal matching pursuit (OMP) algorithm. Here, order-recursive least squares (ORLS) compositions are utilized for balancing of spectral efficiency and computational complexity. In [18], a biomedical signal restoration technique is employed based on compression sensing model for efficient recovery of biomedical signals and enhance quality of restored signals. Here, compression sensing model provide strength to storage and transmission activities as well. In [19], mm-Wave Massive MIMO System is employed for the compression and estimation of CSI feedback based on Multiple-Rate Compressive Sensing techniques. Furthermore, Convolutional Neural Network $(\mathrm{CNN})$ is presented for the enhancement of signal restoration efficiency. In [20], a comprehensive research survey is conducted for proficient resource allocation in ultra-dense mediums like Massive MIMO \& mm-Wave networks, Massive IoT devices and D2D networks etc. Their research challenges, issues and solutions to mitigate them are discussed briefly. In [21], a mm-Wave massive MIMO system is employed for effective channel state information acquisition. Here, a hybrid analog-digital antenna is presented for understanding the insights of analog and digital beamforming. In [22], mm-Wave Massive MIMO model is introduced for channel state information extraction Using Sparse Channel estimation and for effective downlink transmission of signals.

However, several challenges still remain undiscussed and their practical implementation is quite far away. Therefore, a Channel State Information (CSI)-based Sparse Reconstruction (CSISR) technique is presented in this article to encounter challenges of hybrid mm-WAVE communication system. The proposed CSISR technique make their efforts for avoiding optimization problem and reducing channel overhead present in the conventional algorithms. A detailed mathematical modelling of CSISR technique is presented in the following section.

\section{Modelling of Channel State Information (CSI)-based Sparse Reconstruction (CSISR) technique}

This section discusses about a detailed mathematical modelling of Channel State Information (CSI)-based Sparse Reconstruction (CSISR) technique for the precise estimation of CSI at transmitter and receiver side. The 
efficient mathematical representation of CSISR technique enhances efficiency of mm-WAVE communication system. Here, the proposed CSISR technique utilizes less training samples for CSI estimation with lower computational complexity. The proposed CSISR technique ensure proficient signal reconstruction, signal compression and resource reduction by utilizing sparse nature of biomedical signals. Moreover, the proposed CSISR technique reduces antenna coupling errors and phase disturbances. A joint sparse coding algorithm is presented for the optimization of transmitted and received biomedical signals. This algorithm jointly optimize the common sparse nature of biomedical signals and reduces noise present in these signals.

Assume that a composite hybrid mm-Wave massive MIMO network model is considered for efficient channel state information estimation based on proposed CSISR technique in a multiple consumer system. Here, hybrid mm-Wave massive MIMO model is employed with a Security Control Point (SCP) and Consumer Equipment (CE). The Security Control Point (SCP) is responsible for developing a wireless network in a designated region. The SCP of mm-Wave massive MIMO model consists of $K_{b}$ antennas and $M_{b}$ radio frequency set. The radio frequency set is defined a set of electronic components like mixers, amplifiers, attenuators, filters and detectors. Similarly, Consumer Equipment (CE) consists of $K_{c}$ antennas and $M_{c}$ radio frequency set. The medium present between Consumer Equipment (CE) and Security Control Point (SCP) is considered as frequency-demanding. Here, Orthogonal Frequency Division Multiplexing (OFDM) is easily compatible with mm-Wave massive MIMO model which utilizes $K_{s}$ subcarriers. This subcarriers is defined as the sidebands of radio frequency set and utilized for transmitting additional $K_{a}$ information chains. Here, $K_{a}$ can be defined by following equation (1),

$$
K_{a} \leq \min \left(M_{b}, M_{c}\right)
$$

The hybrid pre-processor and combiner utilized for frequency-demanding mm-Wave massive MIMO model can be defined by following equation (2) for $s^{\text {th }}$ subcarrier which belongs to $N\left(K_{S}\right)$,

$$
T[s]=T_{r s c} T_{b b}[s] \quad \text { and } \quad G[s]=G_{r s c} G_{b b}[s]
$$

Where, $N\left(K_{s}\right)$ represents the index group of multiplicity $K_{s}$ which can be further defined as $N\left(K_{s}\right) \cong$ $\left\{0,1,2, \ldots \ldots \ldots, K_{s}-1\right\}$. Here, $T_{r s c} \in \mathbb{D}^{K_{b} \times M_{b}}$ represents analog pre-processors and $T_{b b}[s] \in \mathbb{D}^{K_{a} \times M_{b}}$ represents digital pre-processors. Similarly, $G_{r s c} \in \mathbb{D}^{K_{c} \times M_{c}}$ analog combiner and $G_{b b}[s] \in \mathbb{D}^{K_{a} \times M_{c}}$ denotes digital combiner. Here, $\mathbb{D}$ is a set of complex numbers. Here, each frequency signal component for both analog pre-processor and combiner encounters similar magnitude of fading while digital pre-processor and combiner experiences different magnitude of fading for each frequency signal component. Consider, a fully linked phase shifting system for both analog pre-processor and combiner. Here, assume that a prior training knowledge of pre-processors and combiners is available for both SCP and CE for the execution of channel state information estimation phase.

Assume that a frequency-demanding medium model is considered using proposed CSISR technique which contains $K_{r}$ cluster with $K_{f}$ beam present in every cluster and a tapped delay distance $K_{i}$. Consequently, the main focus remains on sparse coding and downstream Channel State Information (CSI) estimation. Similarly, the proposed CSISR technique and analysis study can be utilized for upstream CSI as well. The $l-t h$ tapped delay of the downstream medium between SCP and CE is represented by the following equation (3),

$$
E_{l} \in \mathbb{D}^{K_{c} \times K_{b}}
$$

Where, $l \in N\left(K_{i}\right)$ and $E_{l}$ is further defined by the following equation (4),

$$
E_{l}=\left[\left(K_{b} \cdot K_{c}\right) \cdot\left(K_{r} \cdot K_{f}\right)^{-1}\right]^{1 / 2} \cdot \sum_{j=1}^{K_{r}} \sum_{p=1}^{K_{f}} \delta_{j, p} q_{c s}\left(l X_{a}-Y_{j}\right)\left(\mathbb{G}_{V} \Upsilon_{V} \varphi_{V}\left(\Theta_{j, p}\right)\right)\left(\mathbb{G}_{X} \Upsilon_{X} \varphi_{X}\left(\phi_{j, p}\right)\right)^{*}
$$

Where, $q_{c s}(Y)$ represents a fixed finite signal frequency limited operation with all filtering influence considered at $Y$. Here, network sampling time is denoted by $X_{a}$ and $\delta_{j, p} \in \mathbb{D}$ represents a complex yield. Moreover, $\Theta_{j, p}$ and $\phi_{j, p}$ lies in the interval $[-\pi, \pi)$ i.e. $\Theta_{j, p}, \phi_{j, p} \in[-\pi, \pi)$. Here, $\Theta_{j, p}$ is defined as the approximate signal arrival angle and $\phi_{j, p}$ is defined as the approximate signal egress angle of the $p-t h$ beam in $j-t h$ cluster. The approximate signal arrival angle can be calculated by measuring different of arrival time between all the signals of an antenna array whereas the approximate signal egress angle can be evaluated by measuring phase delay between all the departed signals of an antenna array elements from a particular location. 
Furthermore, $\varphi_{V}\left(\Theta_{j, p}\right) \in \mathbb{D}^{K_{C} \times 1}$ represents reaction vectors of antenna array elements at CE whereas $\varphi_{X}\left(\phi_{j, p}\right) \in \mathbb{D}^{K_{b} \times 1}$ represents reaction vectors of antenna array elements at SCP. The reaction vectors are largely depends upon particular configurations of antenna array elements due to design errors. For example, a rectilinear antenna array element is considered with a stable and perfect antenna spacing $l$ in place of considering an ideal and steady linear array elements then $\varphi_{V}(\Theta)$ can be defined as,

$$
\varphi_{V}(\Theta) \triangleq\left(K_{c}^{1 / 2}\right)^{-1}\left[\begin{array}{c}
\left.1, e^{-w 2 \pi\left(l+\alpha_{c, 1}\right) \cdot(\beta \sin (\Theta))^{-1}}, e^{-w 2 \pi\left(2 l+\alpha_{c, 2}\right) \cdot(\beta \sin (\Theta))^{-1}}, \ldots . .\right) \\
e^{-w 2 \pi\left(\left(K_{c}-1\right) l+\alpha_{c, K_{C}-1}\right) \cdot(\beta \sin (\Theta))^{-1}}
\end{array}\right]
$$

Where, $\beta$ is defined as the wavelength of carrier signals and the fluctuations in the antenna spacing $l$ between receiver antenna array elements can be defined as $\alpha_{c, 1}, \ldots \ldots \ldots \ldots \alpha_{c, K_{c}-1}$. Moreover, $\mathbb{G}_{X} \in \mathbb{D}^{K_{b} \times K_{b}}$ represents correlative coupling matrix for sender array elements whereas $\mathbb{G}_{V} \in \mathbb{D}^{K_{c} \times K_{c}}$ represents correlative coupling matrix for collector array elements. These correlative coupling matrix shows the undesirable energy exchange between array elements. Furthermore, $\Upsilon_{X} \in \mathbb{D}^{K_{b} \times K_{b}}$ and $\Upsilon_{V} \in \mathbb{D}^{K_{c} \times K_{c}}$ represents phase disturbance matrix and antenna yield respectively. Then,

$$
\Upsilon_{V} \triangleq \operatorname{diag}\left\{z_{c}, 1^{e^{w \sigma_{c, 1}}}, z_{c}, 2^{e^{w \sigma_{c, 2}}}, \ldots \ldots \ldots, z_{c}, K_{c}^{e^{w \sigma_{c, K}}}\right\}
$$

Where, $\left\{z_{c, n}\right\}_{n=1}^{K_{c}}$ is defined as the receiver yield disturbances formalized to an ideal amplitude whereas $\left\{\sigma_{c, n}\right\}_{n=1}^{K_{c}}$ represents extra receiver phase disturbances. The main reason of antenna yield and phase disturbances are hardware design issues. A sloped matrix $K_{r} K_{f} \times K_{r} K_{f}$ that consists of medium coefficients can be determined by following equation (7),

$$
\epsilon_{l} \triangleq\left[\left(K_{b} \cdot K_{c}\right) \cdot\left(K_{r} \cdot K_{f}\right)^{-1}\right]^{\frac{1}{2}} \operatorname{diag}\left\{\delta_{1,1} q_{c s}\left(l X_{a}-Y_{1}\right), \ldots \ldots, \delta_{K_{r} K_{f}} q_{c s}\left(l X_{a}-Y_{K_{r}}\right)\right\}
$$

Then, equation (4) is further classified by the following equation (8) as,

$$
E_{l}=\mathbb{G}_{V} \Upsilon_{V} H_{V} \epsilon_{l} \mathbb{G}_{X}{ }^{*} \Upsilon_{X}{ }^{*} H_{X}{ }^{*}
$$

Where, $H_{V} \triangleq\left[\varphi_{V}\left(\Theta_{1,1}\right), \ldots \ldots, \varphi_{V}\left(\Theta_{K_{r}, K_{f}}\right)\right]$ represents reaction vectors of collector antenna array elements calculated at the real signal arrival angle whereas $H_{X} \triangleq\left[\varphi_{X}\left(\phi_{1,1}\right), \ldots \ldots, \varphi_{X}\left(\phi_{K_{r}, K_{f}}\right)\right]$ represents reaction vectors of sender antenna array elements calculated at the real signal egress angle. With the help of proposed CSISR technique the sparsity in the medium matrix can be exploited. For that equation (8) can be further classified using elongated medium model as,

$$
E_{l} \cong \mathbb{G}_{V} \gamma_{V} H_{V}^{\mathbb{W}} \epsilon_{l}^{\mathbb{W}}\left(H_{X}^{\mathbb{W}}\right)^{*} \gamma_{X}^{*} \mathbb{G}_{X}{ }^{*}
$$

Here, steering matrices $H_{V}^{\mathbb{W}} \in \mathbb{D}^{K_{c} \times U_{c}}$ and $H_{X}^{\mathbb{W}} \in \mathbb{D}^{K_{b} \times U_{b}}$ optimizes $H_{V}$ and $H_{X}$ respectively whereas $\epsilon_{l}$ is optimized by $\epsilon_{l}^{\mathbb{W}} \in \mathbb{D}^{U_{b} \times U_{c}}$. Furthermore, $H_{V}^{\mathbb{W}}$ represents the reaction vectors of collector antenna array elements calculated based on $U_{c}$ approximated angles for signal arrival angle whereas $H_{X}^{\mathbb{W}}$ represents the reaction vectors of sender antenna array elements calculated based on $U_{b}$ approximated angles for signal egress angle. Both $H_{V}^{\mathbb{W}}$ and $H_{X}^{\mathbb{W}}$ lies in the interval $[-\pi, \pi)$ and $\epsilon_{l}^{\mathbb{W}}$ consists of trajectory yield of these disjoined approximated signal arrival angle and signal egress angle at non-null array elements. It is assumed that the prior knowledge of the coefficients $\mathbb{G}_{V}, \mathbb{G}_{X}, \gamma_{V}$ and $\gamma_{X}$ present in the equation (9) are available to understand the sparse nature of signals using proposed CSISR technique based on the sparse coding algorithm. Consider that each correlative coupling matrix and phase disturbance matrix are identity matrix whereas antenna spacing matrix is a zero matrix. Then, $\mathbb{G}_{V} Y_{V} H_{V}^{\mathbb{W}}$ and $\mathbb{G}_{X} Y_{X} H_{X}^{\mathbb{W}}$ are replaced by two basic steering matrices $\mathbb{S}_{V}$ and $\mathbb{S}_{X}$ without any limitation of array element design. Therefore, these steering matrices are employed to any random antenna configuration. Then, equation (8) can be further decomposed into following equation (10) as,

$$
E_{l} \cong \mathbb{S}_{V} \aleph_{l} \mathbb{S}_{X}^{*}
$$

Where, $\quad \mathbb{S}_{V} \triangleq\left[\mathbb{S}_{V, 0}, \mathbb{S}_{V, 1}, \ldots \ldots \ldots \mathbb{S}_{V, P_{c}-1}\right] \in \mathbb{D}^{K_{c} \times P_{c}} \quad$ and $\quad \mathbb{S}_{X} \triangleq\left[\mathbb{S}_{X, 0}, \mathbb{S}_{X, 1}, \ldots \ldots \ldots \mathbb{S}_{X, P_{b}-1}\right] \in \mathbb{D}^{K_{b} \times P_{b}}$ represents finest receiver and transmission steering matrices to be evaluated whereas $\aleph_{l} \in \mathbb{D}^{P_{c} \times P_{b}}$ is a sparse 
medium matrices with some non-null array elements same as $\epsilon_{l}^{\mathbb{W}}$ shown in equation (9). To discard uncertainties between steering matrices and medium matrices, the columns of steering matrices can be optimized by following equations,

$$
\left\|\mathbb{S}_{V, p_{c}}\right\|_{2}=1, \forall p_{c} \in N\left(P_{c}\right) \text { and }\left\|\mathbb{S}_{X, p_{b}}\right\|_{2}=1, \forall p_{b} \in N\left(P_{b}\right)
$$

Where, $P_{c} \geq K_{c}$ and $P_{b} \geq K_{b}$ are the number of columns for every steering matrices. Here, it is anticipated that the novel medium matrix $\aleph_{l}$ contains higher sparsity than its counterparts $\epsilon_{l}^{\mathbb{W}}$ due to less uncertainties present in normalized steering matrices and easily adjustable to various conditions of medium matrix.

The frequency domain of medium matrices at the $s-t h$ subcarrier where $s \in N\left(M_{s}\right)$ for the configured medium matrix of equation (10) can be defined as,

$$
E[s]=\sum_{l=0}^{K_{i}-1} E_{l} e^{-w \frac{2 \pi s l}{K_{S}}}=\mathbb{G}_{V} \Upsilon_{V} H_{V}\left(\sum_{l=0}^{K_{i}-1} \epsilon_{l} e^{-w \frac{2 \pi s l}{K_{S}}}\right) \mathbb{G}_{X}{ }^{*} \Upsilon_{X}{ }^{*} H_{X}{ }^{*} \cong \mathbb{S}_{V}\left(\sum_{l=0}^{K_{i}-1} \aleph_{l} e^{-w \frac{2 \pi s l}{K_{S}}}\right) \mathbb{S}_{X}^{*}
$$

Where, $\left(\sum_{l=0}^{K_{i}-1} \epsilon_{l} e^{-w \frac{2 \pi s l}{K_{S}}}\right)=\epsilon[s]$ and $\left(\sum_{l=0}^{K_{i}-1} \aleph_{l} e^{-w \frac{2 \pi s l}{K_{S}}}\right)=\aleph[s]$ represents the medium yield in frequency domain. Consider that $\mathbb{S}_{V}, \mathbb{S}_{X}, \mathbb{G}_{V}, \mathbb{G}_{X}, H_{V}, H_{X}, \Upsilon_{V}, \Upsilon_{X}$ all are frequency independent. Then,

$$
\operatorname{vec}(E[s])=\left(\left(\overline{\mathbb{G}_{X} H_{X} Y_{X}}\right) \otimes\left(\mathbb{G}_{V}, H_{V}, Y_{V}\right)\right) \operatorname{vec}(\epsilon[s]) \cong\left(\overline{\mathbb{S}_{X}} \otimes \mathbb{S}_{V}\right) \operatorname{vec}(\aleph[s])=\xi \tilde{e}[s]
$$

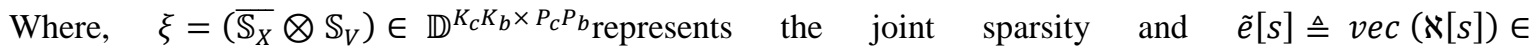
$\mathbb{D}^{P_{c} P_{b} \times 1}$ represents the vectorised steering medium matrices when joint sparsity matrix is utilized to evaluate $\operatorname{vec}(E[s])$.

From equation (9) and (10), the collected signal at CE for the $s-t h$ subcarrier is evaluated as,

$$
\Psi[s]=G_{b b}^{*}[s] G_{r s c}^{*} E[s] T_{r s c} T_{b b}[s] a[s]+G_{b b}^{*}[s] G_{r s c}^{*} \Omega[s]
$$

Where, $a[s] \in \mathbb{D}^{M_{a} \times 1}$ represents the broadcasted signal the sender side for the $s-t h$ subcarrier and $\Omega[s] \approx \tau \Gamma\left(0, \rho^{2} N_{K_{C}}\right)$ represents Gaussian disturbance vector with variance $\rho^{2}$. Consider that at the time of channel state information estimation and sparse coding implementation stage, CE and SCP utilizes pre-processor $T_{\varrho} \in \mathbb{D}^{K_{b} \times M_{b}}$ and combiner $G_{\varrho} \in \mathbb{D}^{K_{c} \times M_{c}}$ of similar frequency for each subcarrier signal in the $\varrho-$ th OFDM. Then, the broadcasted signal is defined as,

$$
\mathbb{Z}\left\{a_{\varrho}[s] a_{\varrho}^{*}[s]\right\}=\left(\mathbb{E} / K_{a}\right) N_{K_{a}}
$$

Where, $\mathbb{E}$ denotes total limiting energy then SNR is defined as $S N R=\mathbb{E} .\left(\rho^{2}\right)^{-1}$. To formularize channel state information estimation and sparse coding implementation, the broadcasted signal is further decomposed as,

$$
a_{\varrho}[s]=\varsigma_{\varrho} b_{\varrho}[s]
$$

Where, $\varsigma_{\varrho} \in \mathbb{D}^{M_{b} \times 1}$ is a frequency set of similar magnitudes and $b_{\varrho}[s]$ is a complex exponential in time. Then, training series can be evaluated using sparse coding based on the additional signal series $c_{k}[s]$. Where, $=0,1,2 \ldots \ldots$. Then, the training series $b_{\varrho}[s]$ for a propagation factor $K_{\beth}$ can be defined as,

$$
b_{\varrho}[s]=c_{\left\lfloor\frac{\varrho}{K_{\beth}}\right\rfloor}[s]
$$

Then, equation (17) can be further discomposed as,

$$
b_{\varrho}[s]=c_{0}[s], \ldots \ldots c_{0}[s], c_{1}[s], \ldots \ldots c_{1}[s], \ldots .
$$

Then, after joining the training series at collector side for the $s-t h$ subcarrier in the $\varrho-t h$ OFDM training signal is evaluated as, 


$$
\Psi_{\varrho}[s]=G_{\varrho}^{*} E[s] T_{\varrho} S_{\varrho} b_{\varrho}[s]+G_{\varrho}^{*} \Omega_{\varrho}[s]
$$

With the help of sparse coding for efficient signal reconstruction the collected signal $\Psi_{\varrho}[s]$ is multiplied $\operatorname{with}\left(b_{\varrho}[s]\right)^{-1}$. Then,

$$
\widetilde{\Psi}_{\varrho}[s] \triangleq \operatorname{vec}\left(\left(b_{\varrho}[s]\right)^{-1} \Psi_{\varrho}[s]\right)=\left(T_{\varrho}^{X} S_{\varrho}^{X} \otimes G_{\varrho}^{*}\right) \operatorname{vec}(E[s])+\widetilde{\Omega}_{\varrho}[s]
$$

Where, $\widetilde{\Omega}_{\varrho}[s] \triangleq \operatorname{vec}\left(\left(b_{\varrho}[s]\right)^{-1} G_{\varrho}^{*} \Omega_{\varrho}[s]\right)$. Then, from equation from equation (13) and (20),

$$
\widetilde{\Psi}_{\varrho}[s] \cong\left(T_{\varrho}^{X} \varsigma_{\varrho}^{X} \otimes G_{\varrho}^{*}\right)\left(\overline{\mathbb{S}_{X}} \otimes \mathbb{S}_{V}\right) \operatorname{vec}(\aleph[s])+\widetilde{\Omega}_{\varrho}[s]=\lambda_{\varrho} \xi \tilde{e}[s]+\widetilde{\Omega}_{\varrho}[s]
$$

Where, $\lambda_{\varrho} \triangleq\left(T_{\varrho}^{X} S_{\varrho}^{X} \otimes G_{\varrho}^{*}\right) \in \mathbb{D}^{M_{c} \times K_{c} K_{b}}$ represents an observation matrix which depends on upon preprocessors and combiners for $\varrho-t h \mathrm{OFDM}$ and $\lambda_{\varrho} \xi$ is a quantification matrix used for sparse signal reconstruction. Furthermore, take out average of collected signals for noise free sparse signal reconstruction,

$$
\widetilde{\Psi}_{\text {avg }, n}[s]=\sum_{\varrho=0}^{K_{\beth}-1} \widetilde{\Psi}_{n K_{\beth}+\varrho}[s] / K_{\beth} \text { for } n=0,1,2 \ldots .
$$

Then, from the average quantification the observation matrix is evaluated at the $s-t h$ subcarrier on the collector side to formulate received signals,

$$
\left[\widetilde{\Psi}^{X}{ }_{a v g, 0}[s], \ldots ., \widetilde{\Psi}^{X}{ }_{a v g, \Delta-1}[s]\right]^{X}=\left[\lambda_{0}^{X}, \ldots \ldots \lambda_{\Delta-1}^{X}\right]^{X} \xi \tilde{e}[s]+\left[\widetilde{\Omega}^{X}{ }_{a v g, 0}[s], \ldots \ldots, \widetilde{\Omega}^{X}{ }_{a v g, \Delta-1}[s]\right]^{X}
$$

Where,

$$
\widetilde{\Psi}[s] \in \mathbb{D}^{\Delta M_{c} \times 1}, \lambda \in \mathbb{D}^{\Delta M_{c} \times K_{c} K_{b}} \text { and } \widetilde{\Omega}[s] \in \mathbb{D}^{\Delta M_{c} \times 1}
$$

\section{Result and Discussions:}

This section discusses about the performance comparison of proposed Channel State Information (CSI)-based Sparse Reconstruction (CSISR) technique with other state-of-art-techniques in terms of average spectral efficiency, power consumption and energy efficiency. Furthermore, the hybrid mm-Wave massive MIMO system is considered for the detailed investigation of performance results. The efficiency of the mm-Wave massive MIMO system is heavily depends upon the effective estimation of Channel State Information (CSI). The proposed CSISR technique utilizes minimum training samples for effective signal reconstruction, signal compression and resource reduction under low SNR conditions as well by exploiting sparse nature of biomedical signals. The sparse coding algorithm exploits common sparse nature of subcarriers to reduce antenna disturbances and antenna coupling errors. The conventional state-of-art algorithms like Orthogonal Matching Pursuit (OMP) algorithm consists of optimization problem and channel overhead issues which are mitigated using proposed CSISR technique. The performance throughput of proposed sparse coding algorithm is measured against conventional algorithms considering power consumption, Normalized Mean Square Error (NMSE) and efficiency of the mm-Wave MIMO system. All the performance results are simulated using MATLAB ${ }^{T M}$.

In this article, performance results like average spectral efficiency, power consumption, NMSE and energy efficiency are highlighted for proposed CSISR technique. The Monte-Carlo realizations are averaged at 100 to simulate performance results. A significant reduction in computational complexity is observed using the proposed CSISR technique in contrast to conventional techniques. It is assumed that there is an ideal synchronization take place between broadcaster and collector sides for the effective estimation of CSI. The simulation parameters considered for performance evaluation is presented in Table 1. Furthermore, the proposed CSISR technique is compared against several state-of-art-techniques like Digital Beamforming [24], Conventional Analog Beamforming with Zadoff - Chu $(Z C)$ [25] and Conventional Analog Beamforming with Phase Shifter (PS) [26] and Conventional Analog Beamforming-2 with Phase Shifter (PS) for Average Spectral Efficiency (ASE) [27] considering different scenarios and parameters as shown in Figure 1, Figure 2 and Figure 3. Here, Figure 1-3 demonstrates the function of RX RF chains considering different mm-Wave massive MIMO system parameters at Signal to Noise Ratio $(\mathrm{SNR})=15 d B$. It is evident from Figure 1-3 that the signals which are lost due to non-orthogonality of conventional analog joint matrix are effectively recovered 
using proposed CSISR technique. It is also observed that the proposed CSISR technique shows smoother curve for low to mid number of RF chains than any other state-of-art-techniques which implies best results for effective channel estimation and channel overhead reduction for a cellular network among all the compared techniques considering different scenarios.

Table 1. Simulation Parameters for Performance Evaluation

\begin{tabular}{|l|l|}
\hline Simulation Parameter & Parameter Value \\
\hline Channel Delay Tap & 4 \\
\hline Monte-Carlo realizations & 100 \\
\hline Signal To Noise Ratio (SNR) (dB) & 15 \\
\hline Number of Frames & 40 \\
\hline Number of antenna elements at Transmitter & 16 \\
\hline Number of Propagation path per channel delay tap & 6 \\
\hline Antenna Array Characteristics & Uniform Linear Array (ULA) \\
\hline
\end{tabular}

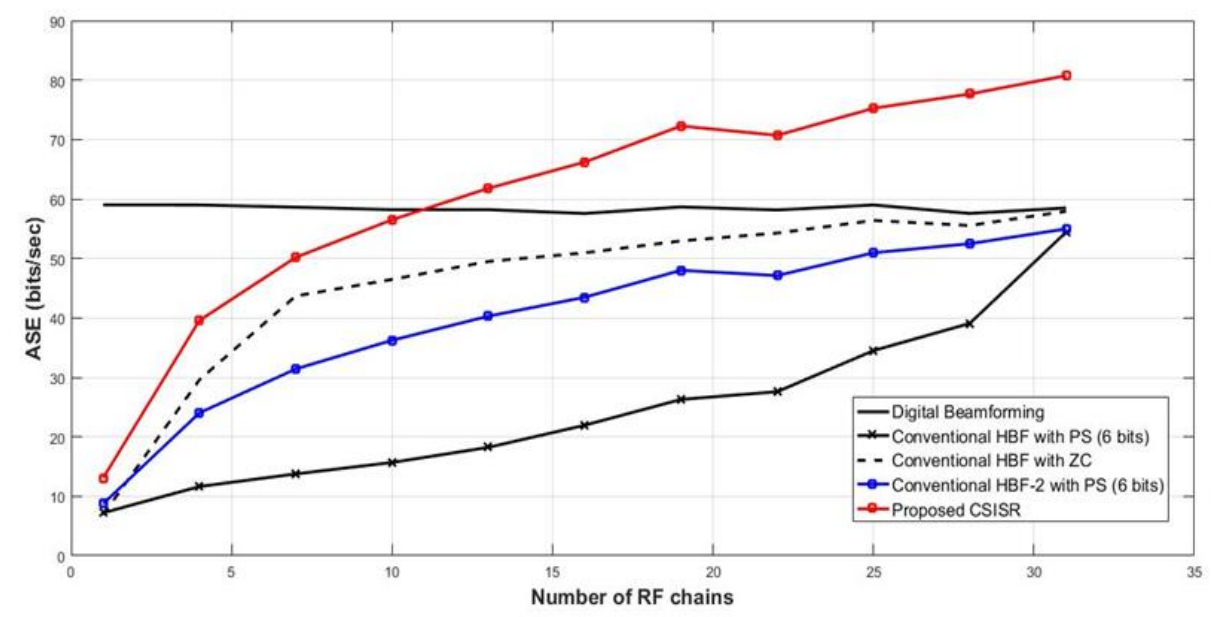

Figure 1. ASE as a function of the number of RF chains considering antenna elements at receiver $=32$ and number of outputs $=32$ and using simulation parameters shown in Table 1

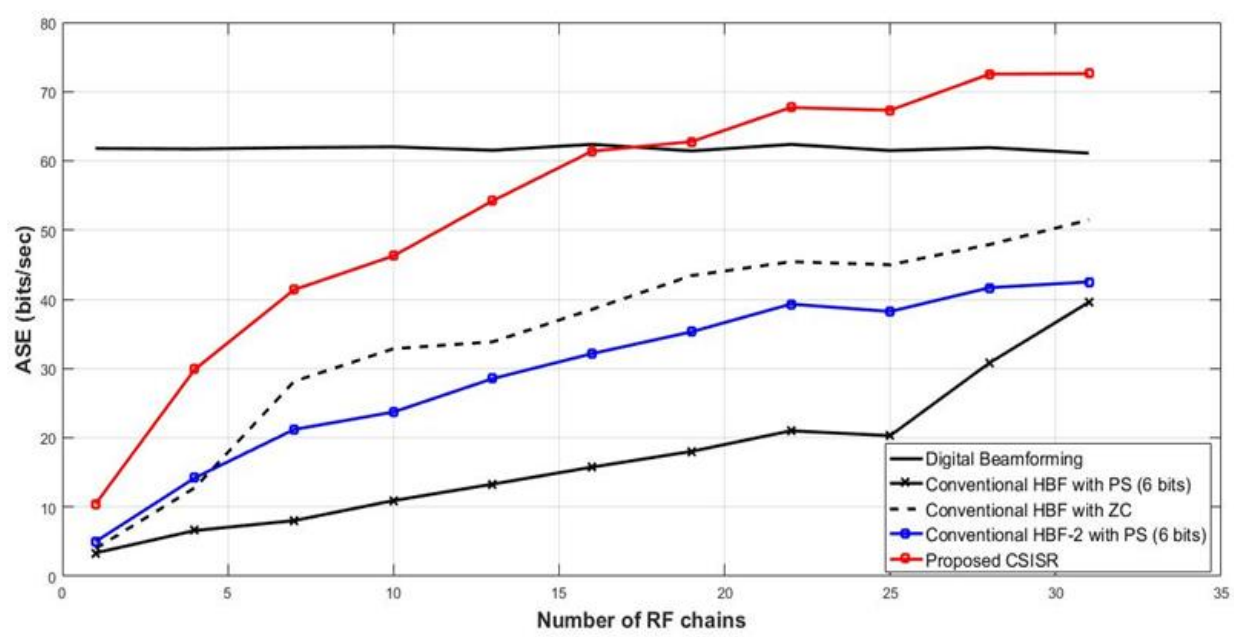

Figure 2. ASE as a function of the number of RF chains considering antenna elements at receiver $=64$ and number of outputs $=32$ and using simulation parameters shown in Table 1 


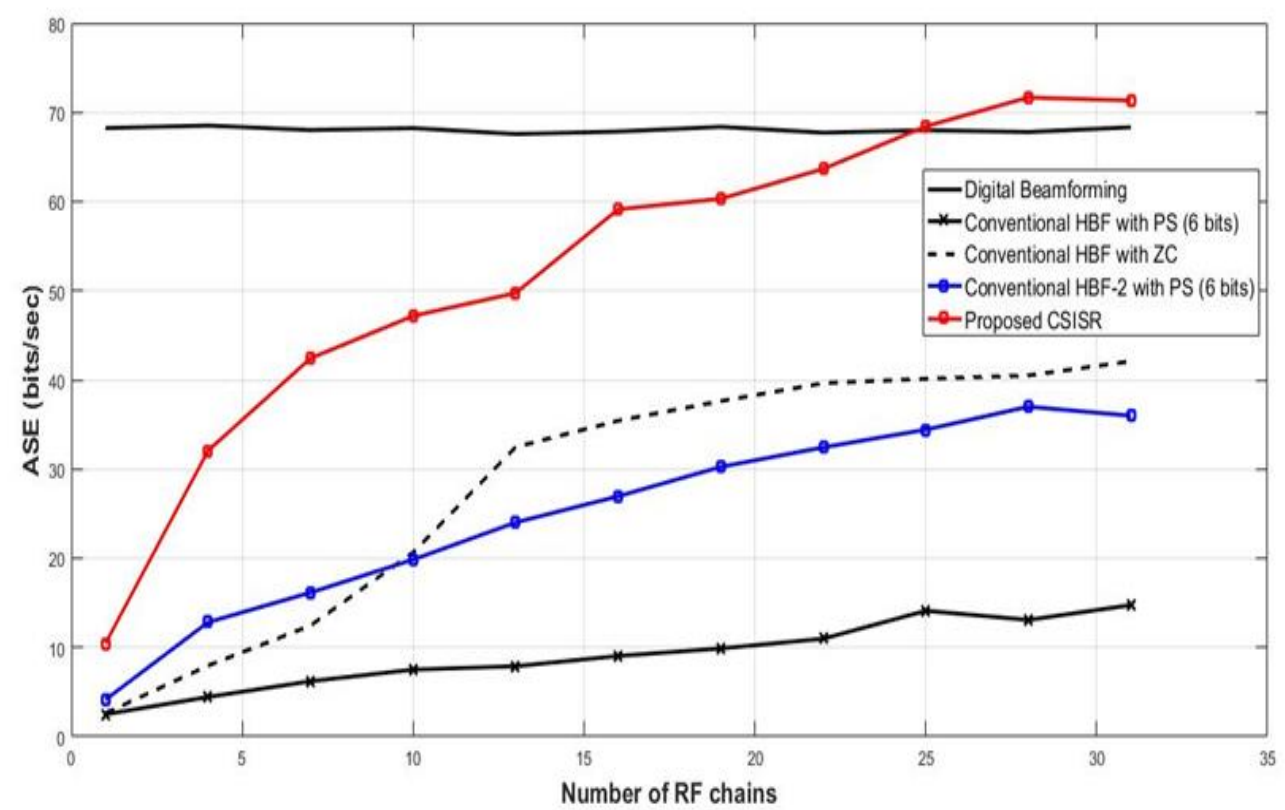

Figure 3. ASE as a function of the number of RF chains considering antenna elements at receiver $=128$ and number of outputs $=64$ and using simulation parameters shown in Table 1

Then, a detailed study of power consumption in the mm-Wave MIMO system is conducted and their performance result are presented in Figure 4 and Figure 5. Here, Figure 4 demonstrates required power for the mm-Wave MIMO system in $m W$ as a function of RX RF chains. The simulation results are obtained by considering ideal power parameters used in [23] for state-of-art-techniques like Digital Beamforming, Conventional Analog Beamforming with Zadoff - Chu (ZC), Conventional Analog Beamforming with Phase Shifter (PS) and Conventional Analog Beamforming-2 with Phase Shifter (PS). It is evident from Figure 4 that power consumption in the mm-Wave MIMO system using proposed CSISR technique is minimum than compare other state-of-art-techniques.

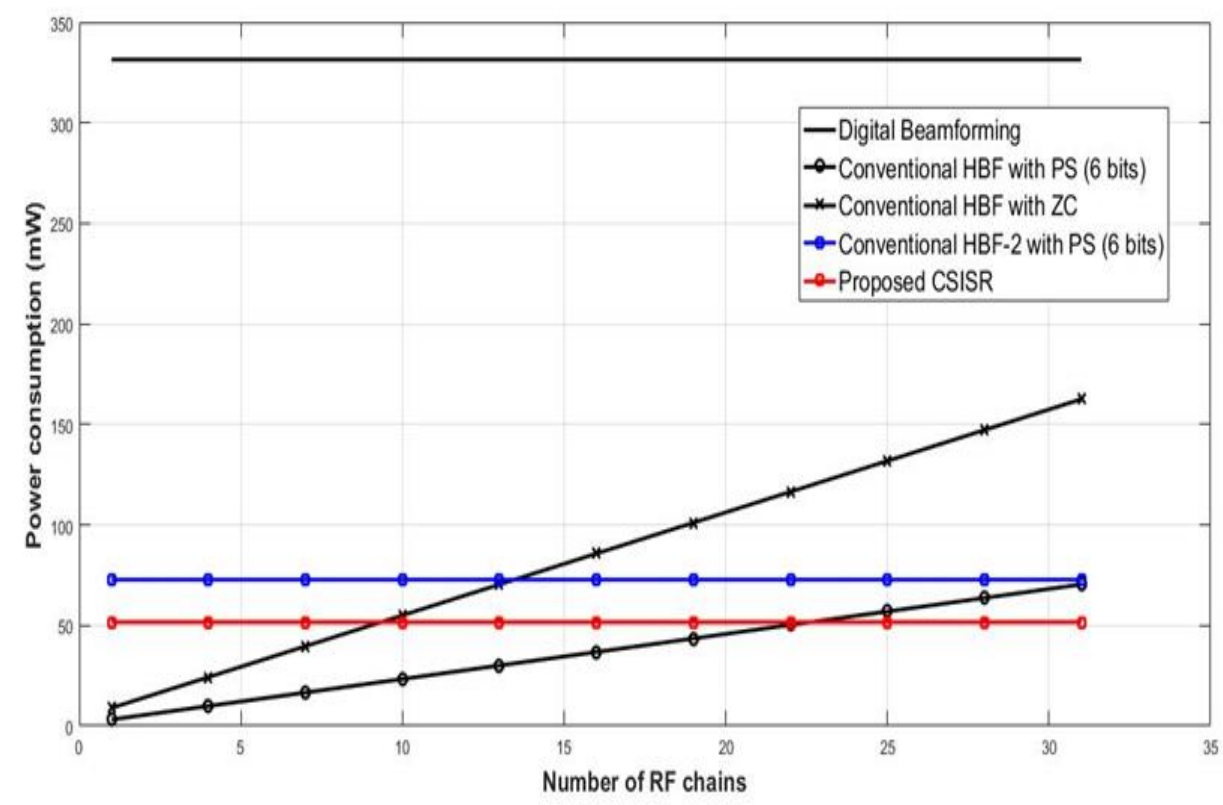

Figure 4. Power Consumption with respect to the number of RF chains considering antenna elements at receiver $=64$ and number of outputs $=32$ and using simulation parameters shown in Table 1 


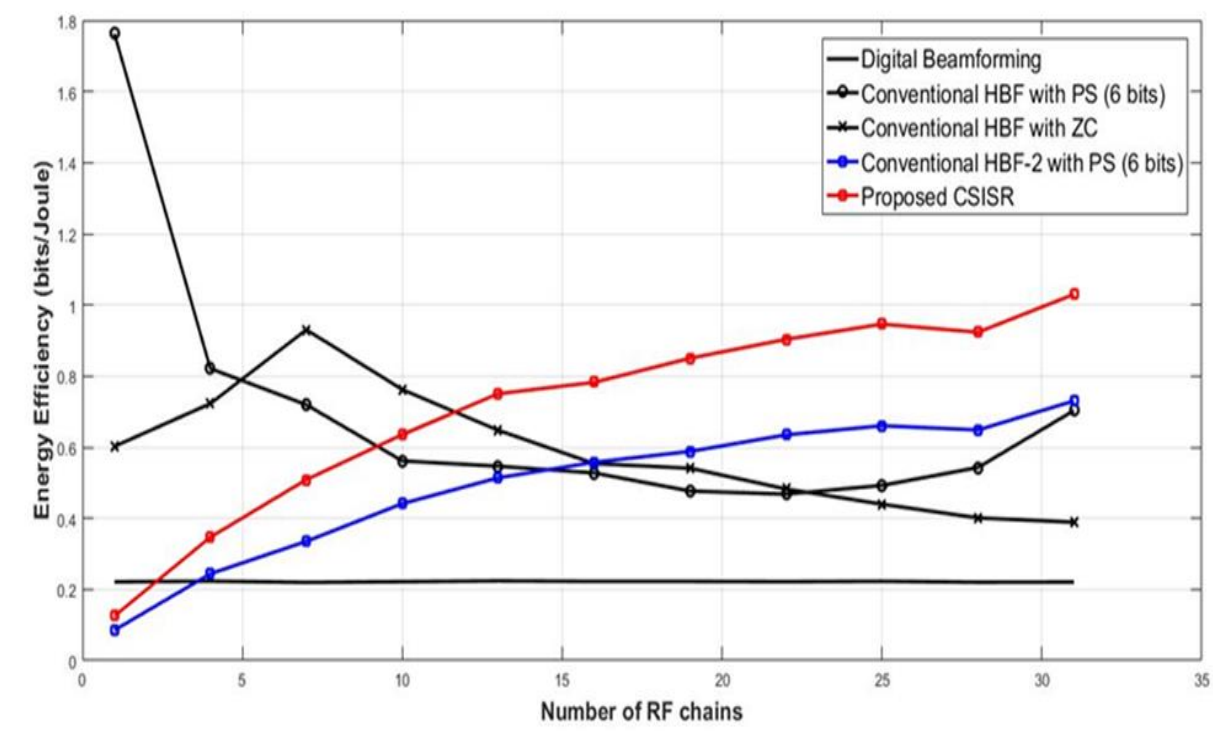

Figure 5. Energy Efficiency with respect to the number of RF chains considering antenna elements at receiver $=64$ and number of outputs $=32$ and using simulation parameters shown in Table 1

Furthermore, the power requirement details is analysed in term of energy efficiency as well for the mm-Wave MIMO system. Here, Figure 5 demonstrates energy efficiency for the mm-Wave MIMO system in $\frac{\text { Mbits }}{\text { Joule }}$ as a function of RX RF chains and energy efficiency is defined by following equation,

$$
E E \triangleq A S P .(P C)^{-1}(\text { Mbits/Joule })
$$

Where, $P C$ represents power consumption and $A S P$ is defined as the average spectral efficiency. The proposed CSISR technique outperforms all the state-of-art-techniques in terms of average spectral efficiency, energy efficiency as well as power consumption.

\section{Conclusion}

In this article, Channel State Information (CSI)-based Sparse Reconstruction (CSISR) technique is presented to evaluate CSI and reduce channel overhead of mm-WAVE communication system using biomedical signal transmission for the efficiency enhancement of future 5G cellular network. A detailed mathematical modelling for the CSI estimation and channel overhead reduction is presented. The proposed CSISR technique ensure proficient signal reconstruction, signal compression and resource reduction by utilizing sparse nature of biomedical signals. Furthermore, a composite mm-Wave massive MIMO network model is considered for efficient channel state information estimation. A joint sparse coding algorithm is presented to optimize antenna coupling errors and phase disturbances by exploiting common sparse nature of biomedical signals and efficiently reconstruct the original transmitted signals. The hybrid mm-Wave massive MIMO model is employed with a Security Control Point (SCP) and Consumer Equipment (CE). The hybrid pre-processor and combiner utilized for frequency-demanding mm-Wave massive MIMO model using proposed CSISR technique. In simulation results, Figure 1-3 discusses about the lost signal reconstruction considering different performance matrices whereas Figure 4-5 discusses about the power consumption reduction and high energy efficiency achieved using proposed CSISR technique The proposed CSISR technique outperforms all the state-of-art-techniques performance-wise for the performance matrices like average spectral efficiency, energy efficiency as well as power consumption.

\section{References}

1. Dattatreya M Desai, Sanjay H S, Ankitha S, Prithvi B S, Dinesh P A, "Psychoacoustical Differentiation with Regard to the Perception of Pitch - A Comparison Between Musicians and Non-musicians", Journal of Computational \& Theoretical Nanoscience, Vol 17 (9/10), 4506-09, (2020) (DOI: 10.1166/jctn.2020.9096). 
2. Orovic, A. Draganic, N. Lekic, S. Stankovic, "A System for Compressive Sensing Signal Reconstruction," 17th IEEE International Conference on Smart Technologies, IEEE EUROCON 2017, 6th-8th July 2017, Ohrid, Macedonia, 2017

3. S. Stankovic, I. Orovic, LJ. Stankovic, "An Automated Signal Reconstruction Method based on Analysis of Compressive Sensed Signals in Noisy Environment," Signal Processing, vol. 104, Nov 2014, pp. 43 - 50, 2014.

4. A. Draganic, I. Orovic, S. Stankovic, X. Li, Z. Wang, "An approach to classification and undersampling of the interfering wireless signals," Microprocessors and Microsystems, Volume 51, June 2017, Pages 106-113, 2017.

5. Draganic, I. Orovic, S. Stankovic, X. Zhang, X. Wang, "Compressive Sensing Approach in the Table Grape Cold Chain Logistics", 6th Mediterranean Conference on Embedded Computing, MECO, 2017, Bar, Montenegro, 2017.

6. M. Medenica, S. Zukovic, A. Draganic, I. Orovic, S. Stankovic, "Comparison of the algorithms for CS image reconstruction," ETF Journal of Electrical Engineering, 09/2014; 20(1):29-39., 2014

7. T. S. Rappaport, J. N. Murdock, and F. Gutierrez, "State of the art in $60-\mathrm{GHz}$ integrated circuits and systems for wireless communications," Proc. IEEE, vol. 99, no. 8, pp. 1390-1436, Aug. 2011.

8. L. Swindlehurst, E. Ayanoglu, P. Heydari, and F. Capolino, "Millimeter-wave massive MIMO: the next wireless revolution?” IEEE Commun. Mag., vol. 52, no. 9, pp. 56-62, September 2014.

9. X. Li, J. Fang, H. Duan, Z. Chen and H. Li, "Fast Beam Alignment for Millimeter Wave Communications: A Sparse Encoding and Phaseless Decoding Approach," in IEEE Transactions on Signal Processing, vol. 67, no. 17, pp. 4402-4417, 1 Sept.1, 2019, doi: 10.1109/TSP.2019.2929460.

10. K. Anjinappa, Y. Zhou, Y. Yapici, D. Baron and I. Guvenc, "Channel Estimation in mmWave Hybrid MIMO System via Off-Grid Dirichlet Kernels," 2019 IEEE Global Communications Conference (GLOBECOM), Waikoloa, HI, USA, 2019, pp. 1-6, doi: 10.1109/GLOBECOM38437.2019.9013906.

11. Attiah, M.L., Isa, A.A.M., Zakaria, Z. et al. A survey of mmWave user association mechanisms and spectrum sharing approaches: an overview, open issues and challenges, future research trends. Wireless Netw 26, 2487-2514 (2020). https://doi.org/10.1007/s11276-019-01976-x

12. R. W. Heath, Jr., N. Gonz'alez-Prelcic, S. Rangan, W. Roh, and A. M. Sayeed, "An overview of signal processing techniques for millimetre wave MIMO systems," IEEE J. Sel. Topics Signal Process., vol. 10, no. 3, pp. 436-453, Apr. 2016.

13. K. Venugopal, A. Alkhateeb, R. W. Heath Jr., and N. Gonz'alez-Prelcic, "Time-domain channel estimation for wideband millimeter wave systems with hybrid architecture," in Proc. IEEE ICASSP, New Orleans, USA, 2017, pp. 6493-6497.

14. Alkhateeb, O. E. Ayach, G. Leus, and R. W. Heath Jr., "Channel estimation and hybrid precoding for millimeter wave cellular systems," IEEE J. Sel. Topics Signal Process., vol. 8, no. 5, pp. 831-846, Oct. 2014

15. L. Cheng, G. Yue, D. Yu, Y. Liang and S. Li, "Millimeter Wave Time-Varying Channel Estimation via Exploiting Block-Sparse and Low-Rank Structures," in IEEE Access, vol. 7, pp. 123355-123366, 2019, doi: 10.1109/ACCESS.2019.2937628.

16. L. T. Nguyen, J. Kim and B. Shim, "Low-Rank Matrix Completion: A Contemporary Survey," in IEEE Access, vol. 7, pp. 94215-94237, 2019, doi: 10.1109/ACCESS.2019.2928130.

17. Y. Zhang, Y. Huang, X. Qin and P. Zhang, "Low complexity hybrid precoding based on ORLS for mmWave massive MIMO systems," 2018 IEEE Wireless Communications and Networking Conference (WCNC), Barcelona, 2018, pp. 1-6, doi: 10.1109/WCNC.2018.8377060.

18. V. Mandić and I. Martinović, "Biomedical signals reconstruction under the compressive sensing approach," 2018 7th Mediterranean Conference on Embedded Computing (MECO), Budva, 2018, pp. 14, doi: 10.1109/MECO.2018.8405978.

19. J. Guo, C. Wen, S. Jin and G. Y. Li, "Convolutional Neural Network-Based Multiple-Rate Compressive Sensing for Massive MIMO CSI Feedback: Design, Simulation, and Analysis," in IEEE Transactions on Wireless Communications, vol. 19, no. 4, pp. 2827-2840, April 2020, doi: 10.1109/TWC.2020.2968430.

20. Y. Teng, M. Liu, F. R. Yu, V. C. M. Leung, M. Song and Y. Zhang, "Resource Allocation for UltraDense Networks: A Survey, Some Research Issues and Challenges," in IEEE Communications Surveys \& Tutorials, vol. 21, no. 3, pp. 2134-2168, thirdquarter 2019, doi: 10.1109/COMST.2018.2867268.

21. S. Chen, Q. Gao, R. Chen, H. Li, S. Sun and Z. Liu, "A CSI acquisition approach for mmWave massive MIMO," in China Communications, vol. 16, no. 9, pp. 1-14, Sept. 2019, doi: 10.23919/JCC.2019.09.001.

22. Lv, J. Lin and Z. Yang, "CSI Calibration for Precoding in mmWave Massive MIMO Downlink Transmission Using Sparse Channel Prediction," in IEEE Access, vol. 8, pp. 154382-154389, 2020, doi: 10.1109/ACCESS.2020.3017787. 
23. R. M’endez-Rial, C. Rusu, N. Gonz'alez-Prelcic, A. Alkhateeb, and R. W. Heath, Jr., "Hybrid MIMO architectures for millimeter wave communications: Phase shifters or switches?" IEEE Access, vol. 4, pp.247-267, Jan. 2016.

24. F. Molisch, V. V. Ratnam, S. Han, Z. Li, S. L. H. Nguyen, L. Li, and K. Haneda, "Hybrid beamforming for massive MIMO: A survey," IEEE Commun. Mag., vol. 55, no. 9, pp. 134-141, Sep. 2017.

25. H. A. V. D. Vorst, Iterative Krylov Methods for Large Linear Systems. Cambridge University Press, 2003.

26. V. Venkateswaran and A.-J. van der Veen, "Analog beamforming in MIMO communications with phase shift networks and online channel estimation," IEEE Trans. Signal Process., vol. 58, no. 8, pp. 4131-4143,Aug. 2010.

27. E. Vlachos, G. C. Alexandropoulos and J. Thompson, "Wideband MIMO Channel Estimation for Hybrid Beamforming Millimeter Wave Systems via Random Spatial Sampling," in IEEE Journal of Selected Topics in Signal Processing, vol. 13, no. 5, pp. 1136-1150, Sept. 2019, doi: 10.1109/JSTSP.2019.2937633. 